\title{
A NOTE ON THE EQUILIBRIUM POINT OF THE GREEN'S FUNCTION FOR AN ANNULUS
}

\section{DEBORAH M. HICKEY}

1. Introduction. In a previous paper* the motion of the equilibrium point of the Green's function for a plane annular region was studied as the pole was shifted along a radius in the neighborhood of the geometric mean circle $C_{0} . \dagger$ The expression for $d r / d r_{0}$ on $C_{0}, r$ being the distance of the equilibrium point from the center of the circles, $r_{0}$ that of the pole, is $-F_{r_{0}} / F_{r}$, where

$$
\begin{aligned}
& F_{r_{0}}=\frac{\partial F}{\partial r_{0}}=-\frac{2}{R}\left[\frac{1}{2 \log R}-\frac{1}{8}+\sum_{m=1}^{\infty} \frac{(-1)^{m} m}{R^{m}-1}\right], \\
& F_{r}=\frac{\partial F}{\partial r}=-\frac{2}{R}\left[\frac{1}{8}+\sum_{m=1}^{\infty} \frac{(-1)^{m} m}{R^{m}+1}\right] .
\end{aligned}
$$

In these formulas 1 and $R$ are the radii of the inner and outer circular boundaries of the region. It was shown by an application of a theorem of Schlömilch $\ddagger$ that $F_{r_{0}}$ does not vanish on $C_{0}$.

In this article this result and others are obtained by a method which seems better adapted to the problem. $\S$

It is noticed that the function

$$
f(z)=\frac{\pi}{\sin \pi z} \frac{z}{e^{a z}-1}, \quad a=\log R,
$$

* D. M. Hickey, The equilibrium point of Green's function for an annular region, Annals of Mathematics, vol. 30 (1929), pp. 373-383.

$\dagger$ The Green's function for this region may be written in the form

$$
\begin{aligned}
g\left(M, M_{0}\right) & =\log \frac{1}{M M_{0}}+\frac{1}{\log R}\left[\log R \log r_{0}-\log r \log r_{0} / R\right] \\
& -\sum_{m=1}^{\infty} \frac{1}{m} \frac{\cos m\left(\theta-\theta_{0}\right)}{R^{2 m}-1}\left\{r^{m}\left[r_{0}^{m}-r_{0}^{-m}\right]+r^{-m}\left[\left(\frac{R^{2}}{r_{0}}\right)^{m}-r_{0}^{m}\right]\right\} .
\end{aligned}
$$

We take $F\left(r, r_{0}\right)=\partial g / \partial r$ for $r=r_{0}=R^{1 / 2}$ and $\theta-\theta_{0}=\pi$.

$\ddagger \ddot{U b e r}$ einige unendliche Reihen, Zeitschrift für Mathematik und Physik, vol. 23 (1878), p. 132.

$\S$ The suggestion that the method of contour integration and the theory of residues might prove useful was given by A. J. Maria. 
where $R$ is a real number greater than 1, has the sum of residues $\sum_{m=1}^{n}(-1)^{m} m /\left(R^{m}-1\right)$ within a suitably selected contour containing as singularities only the poles $1,2, \cdots, n$ of $f(z)$. It turns out by an integration around a contour and certain limiting processes that an expression for $F_{r_{0}}$ on $C_{0}$ is found which is a series of positive terms. The same method applied to a suitably chosen function $f(z)$ yields for the value of $F_{r}$ a series of negative terms. These values show that $d r / d r_{0}$ is positive on $C_{0}$. By means of the preceding results it is proved that $d^{2} r / d r_{0}^{2}$ is negative on $C_{0}{ }^{*}$

2. The Evaluation of $F_{r_{0}}$ and $F_{r}$ on $C_{0}$ by Contour Integration. The contour $C_{n}$ chosen for the evaluation of $F_{r_{0}}$ consists of the lines $x_{n}=n+1 / 2, y_{n}= \pm(2 n+1) \pi / a$, semi-circular arcs that lie to the right of the imaginary axis of positive radius $\rho<\pi /(2 a)$ and $<1$ and centers $\pm 2 m \pi i / a,(m=0,1, \cdots, n)$, and the straight line segments of the imaginary axis exterior to these arcs included between the upper and lower $y_{n}$ lines. The function

$$
f(z)=\frac{\pi}{\sin \pi z} \cdot \frac{z}{e^{a z}-1}
$$

is analytic inside and on $C_{n}$ except at the poles $z=1,2, \ldots, n$ of $\pi / \sin \pi z$. Hence the value of the integral $(1 / 2 \pi i) \int_{C_{n}} f(z) d z$, where the contour $C_{n}$ is traced in the counter-clockwise direction, gives $\sum_{m=1}^{n}(-1)^{m} m /\left(e^{a m}-1\right)$, the sum of the residues of $f(z)$ inside $C_{n}$.

Let $L_{n}$ be the straight line segments on the imaginary axis, $K_{n}$ the semi-circular arcs, and $S_{n}$ the remaining part of $C_{n}$. Over $L_{n}$ the integral can easily be put into the form

$$
-\frac{1}{2} \sum_{m=0}^{n-1} \int_{(2(m+1) \pi / a)-\rho}^{(2 m \pi / a)+\rho} \frac{y d y}{\sinh \pi y}-\frac{1}{2} \int_{(2 n+1) \pi / a}^{(2 n \pi / a)+\rho} \frac{y d y}{\sinh \pi y} .
$$

For the evaluation of the integral over the arc of $K_{n}$ with center at $2 m \pi i / a,(m \neq 0)$, a power series development of $f(\boldsymbol{z})$ about this point is used. Evaluated, the integral gives

$$
-\frac{\pi^{2}}{a^{2}} \frac{m}{\sinh \left(2 m \pi^{2} / a\right)}+P_{m}(\rho)
$$

* It is evident that the corresponding results hold for any annulus. 
where $P_{m}(\rho)$ is a power series in $\rho$ with constant term zero. Over the arc with center at the origin the value is found to be $(-1 /(2 a))+P_{0}(\rho)$. Thus integration around $C_{n}$ gives

$$
\begin{aligned}
& \sum_{m=1}^{n} \frac{(-1)^{m} m}{e^{a m}-1}=\frac{1}{2} \sum_{m=0}^{n-1} \int_{(2 m \pi / a)+\rho}^{(2(m+1) \pi / a)-\rho} \frac{y d y}{\sinh \pi y} \\
& \quad+\frac{1}{2} \int_{(2 n \pi / a)+\rho}^{((2 n+1) \pi / a)-\rho} \frac{y d y}{\sinh \pi y}-\frac{1}{2 a} \\
& -\frac{2 \pi^{2}}{a^{2}} \sum_{m=1}^{n} \frac{m}{\sinh \left(2 m \pi^{2} / a\right)}+\sum_{m=-n}^{n} P_{m}(\rho)+\frac{1}{2 \pi i} \int_{S_{n}} f(z) d z .
\end{aligned}
$$

The value of $(1 / 2 \pi i) \int_{C_{n}} f(z) d z$ is clearly the same for any positive $\rho$ less than both $\pi /(2 a)$ and 1. Letting $\rho$ approach zero in (1), we obtain

$$
\begin{aligned}
& \sum_{m=1}^{n} \frac{(-1)^{m} m}{e^{a m}-1}=\frac{1}{2} \int_{0}^{(2 n+1) \pi / a} \frac{y d y}{\sinh \pi y} \\
& \quad-\frac{2 \pi^{2}}{a^{2}} \sum_{m=1}^{n} \frac{m}{\sinh \left(2 m \pi^{2} / a\right)}-\frac{1}{2 a}+\frac{1}{2 \pi i} \int_{S_{n}} f(z) d z .
\end{aligned}
$$

Now let $n$ become infinite. The left member of (2) has as limit the convergent series $\sum_{m=1}^{\infty}(-1)^{m} m /\left(e^{a m}-1\right)$. The first term on the right approaches the definite integral

$$
\frac{1}{2} \int_{0}^{\infty} \frac{y d y}{\sinh \pi y},
$$

which is known to have the value $1 / 8$. The series approaches

$$
-\frac{2 \pi^{2}}{a^{2}} \sum_{m=1}^{\infty} \frac{m}{\sinh \left(2 m \pi^{2} / a\right)} .
$$

The integral over $S_{n}$ has the limit zero.

To prove this last statement consider the modulus of $\int_{S n} f(z) d z$. It can be shown* that over the entire curve $S_{n}$, $|1 / \sin \pi z|$ and $\left|1 /\left(e^{a z}-1\right)\right|$ are bounded independently of $n$.

* That $|1 / \sin \pi z|$ is bounded on $S_{n}$ independently of $n$ is proved essentially by Lindelöf in Théorie des Résidus, 1905, p. 32, footnote. The statement for $\left|1 /\left(e^{a z}-1\right)\right|$ can be proved in the same manner. 
Let $M$ be the greater of these two bounds. Moreover, on the upper and lower $y_{n}$ lines

$$
\left|\frac{1}{\sin \pi z}\right|<\frac{1}{\left.\sinh \left(\left(2_{n+1}\right) \pi^{2}\right) / a\right)},
$$

and on the right-hand boundary $z=(n+1 / 2)+i y$,

$$
\left|\frac{1}{e^{a z}-1}\right|<\frac{1}{e^{a(n+1 / 2)}-1} \text {. }
$$

It then follows easily that

$$
\begin{array}{r}
\left|\int_{S_{n}} f(z) d z\right|<\frac{M k(2 n+1)^{2}}{2 a}\left[\frac{1}{2 \sinh \left((2 n+1) \pi^{2} / a\right)}\right. \\
\left.+\frac{1}{e^{a(n+1 / 2)}-1}\right],
\end{array}
$$

where $k$ is a constant independent of $n$. This is sufficient to prove the statement.

In the limit for $n$ infinite, (2) gives

$$
\frac{1}{2 a}-\frac{1}{8}+\sum_{m=1}^{\infty} \frac{(-1)^{m} m}{e^{a m}-1}=-\frac{2 \pi^{2}}{a^{2}} \sum_{m=1}^{\infty} \frac{m}{\sinh \left(2 m \pi^{2} / a\right)}
$$

The left side of (3), where $a$ is replaced by $\log R$, multiplied by $-2 / R$, is $F_{r_{0}}$. Thus $F_{r_{0}}$ is positive.

For the evaluation of $F_{r}$ let

$$
\frac{\pi}{\sin \pi z} \frac{z}{e^{a z}+1}
$$

be chosen for $f(z)$. Let the contour of integration $C_{n}$ consist of the lines $x_{n}=n+1 / 2, y_{n}= \pm 2 n \pi i / a$, semi-circular arcs to the right of the imaginary axis of radius $\rho<\pi /(2 a)$ and with their centers at the points $\pm(2 m+1) \pi i / a,(m=0,1, \cdots, n)$, and the portions of the imaginary axis exterior to these arcs between the upper and lower $y_{n}$ lines.

Applied to this function over the chosen contour, the method used above yields easily the result

$$
\frac{1}{8}+\sum_{m=1}^{\infty} \frac{(-1)^{m} m}{e^{a m}+1}=\frac{\pi^{2}}{a^{2}} \sum_{m=0}^{\infty} \frac{2 m+1}{\sinh \left((2 m+1) \pi^{2} / a\right)} .
$$


By use of (4) with $\log R=a, F_{r}$ can be written as

$$
-\frac{2 \pi^{2}}{R(\log R)^{2}} \sum_{m=1}^{\infty} \frac{(2 m+1)}{\sinh \left(\frac{(2 m+1) \pi^{2}}{\log R}\right)}
$$

a series of negative terms. With these values for $F_{r_{0}}$ and $F_{r}$, we conclude that $d r / d r_{0}=-F_{r_{0}} / F_{r}$ on $C_{0}$ is positive.

3. The Sign of $d^{2} r / d r_{0}^{2}$ on $C_{0}$. From $d r / d r_{0}=-F_{r_{0}} / F_{r}$, we calculate the second derivative

$$
\frac{d^{2} r}{d r_{0}^{2}}=F_{r}^{-3}\left[2 F_{r_{0}} F_{r} F_{r_{0} r}-F_{r_{0}}^{2} F_{r r}-F_{r}^{2} F_{r_{0} r_{0}}\right] \text {. }
$$

From the general expressions for $F_{r_{0}}$ and $F_{r}$ in terms of $r, r_{0}$, and $R$, the following relations on $C_{0}$ are found to hold

$$
F_{r_{0} r_{0}}=-R^{-1 / 2} F_{r_{0}}, \quad F_{r r}=-3 R^{-1 / 2} F_{r}, \quad F_{r_{0} r}=-R^{-1 / 2} F_{r_{0}} .
$$

A substitution of these values in (5) gives

$$
\frac{d^{2} r}{d r_{0}^{2}}=R^{-1 / 2} F_{r_{0}} F_{r^{-2}}\left[F_{r_{0}}+F_{r}\right] \text {. }
$$

Since $F_{r_{0}}$ and $F_{r}^{2}$ are positive, the sign of $d^{2} r / d r_{0}^{2}$ on $C_{0}$ is that of $F_{r_{0}}+F_{r}$. From the results of the preceding section we have

$$
F_{r_{0}}+F_{r}=\frac{2 \pi^{2}}{R(\log R)^{2}} \sum_{m=1}^{\infty} \frac{(-1)^{m} m}{\sinh \left(\frac{m \pi^{2}}{\log R}\right)}
$$

This alternating series converges to a negative sum since its terms are in absolute value strictly decreasing to zero. This shows that $d^{2} r / d r_{0}^{2}$ is negative on $C_{0}$.

Mississippi Delta State Teachers College 